\title{
Erratum to: Performance Portability Analysis for Real-Time Simulations of Smoke Propagation Using OpenACC
}

\author{
Anne Küsters ${ }^{1(\bowtie)}(\mathbb{D})$, Sandra Wienke ${ }^{2,3}$ (D) , and Lukas Arnold ${ }^{1}$ (D) \\ 1 JSC, Forschungszentrum Jülich GmbH, Wilhelm-Johnen-Straße, \\ 52428 Jülich, Germany \\ a.kuesters@fz-juelich.de \\ 2 IT Center, RWTH Aachen University, Seffenter Weg 23, \\ 52074 Aachen, Germany \\ 3 JARA-HPC, 52074 Aachen, Germany
}

\section{Erratum to:}

Chapter "Performance Portability Analysis for Real-Time Simulations of Smoke Propagation Using OpenACC" in: J.M. Kunkel et al. (Eds.): High Performance Computing, LNCS 10524, https://doi.org/10.1007/978-3-319-67630-2_35

The ORCIDs of the second and third authors were incorrect in the original version of the paper. The ORCIDs have been corrected.

\footnotetext{
The updated online version of this chapter can be found at https://doi.org/10.1007/978-3-319-67630-2_35 